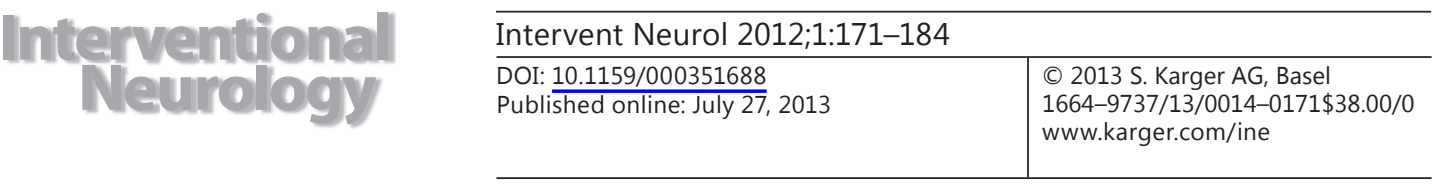

\title{
Chinese Guidelines for Endovascular Management of Ischemic Cerebrovascular Diseases
}

\author{
Xinfeng Liu ${ }^{a}$ Suming Zhang ${ }^{b}$ Ming Liu ${ }^{c}$ Yongjun Wang ${ }^{d}$ Jiang Wu ${ }^{i}$ \\ Qiang Dong ${ }^{j}$ Jinsheng Zeng ${ }^{k}$ Yining Huang ${ }^{f}$ Jian Wu $^{e}$ Yuming $X{ }^{n}$ \\ Kangning Chen ${ }^{\circ}$ Renliang Zhang ${ }^{a}$ Baomin Lig Yajie Liu ${ }^{m}$ Bin Peng ${ }^{h}$ \\ Zhengqi Lu' Huadong Zhou ${ }^{p}$ Xinying Fan ${ }^{a}$ Jie Shuai ${ }^{q}$ Gelin Xu ${ }^{a}{ }^{r}$ \\ a Department of Neurology, Jinling Hospital, Nanjing University School of Medicine, Nanjing, \\ ${ }^{b}$ Department of Neurology, Tongji Hospital, Tongji Medical College Huazhong University \\ of Science and Technology, Wuhan, 'Department of Neurology, West China Hospital, West \\ China School of Medicine, Chengdu, d Department of Neurology, Beijing Tiantan Hospital, \\ and ${ }^{\mathrm{e}}$ Department of Neurology, Xuanwu Hospital, Capital Medical University, ${ }^{f}$ Department \\ of Neurology, Peking University First Hospital, Peking University School of Medicine, \\ ${ }^{9}$ Department of Neurology, No. 301 Hospital of People's Liberation Army, and h' ${ }^{\text {Department }}$ \\ of Neurology, Peking Union Medical College Hospital, Chinese Academy of Medical \\ Sciences and Peking Union Medical College, Beijing, 'Department of Neurology, The First \\ Affiliated Hospital of Jilin University, Bethune Medicine School, Jilin University, Changchun, \\ jDepartment of Neurology, Huashan Hospital, Fudan University School of Medicine, \\ Shanghai, ${ }^{k}$ Department of Neurology, The First Affiliated Hospital of Sun Yat-sen University \\ and 'Department of Neurology, The Third Affiliated Hospital of Sun Yat-sen University, School \\ of Medicine, Sun Yat-sen University, and m Department of Neurology, Zhujiang Hospital, \\ Southern Medical University, Guangzhou, nepartment of Neurology, The First Affiliated \\ Hospital of Zhengzhou University, School of Medicine, Zhengzhou University, Zhengzhou, \\ ${ }^{\circ}$ Department of Neurology, Southwest Hospital, ${ }^{\mathrm{P}}$ Department of Neurology, Daping \\ Hospital, and 9Department of Neurology, Xinqiao Hospital, Third Military Medical University, \\ Chongqing, PR China, 'ron behalf of the Chinese Stroke Society
}

\section{Key Words}

Chinese Stroke Society · Endovascular treatment $\cdot$ Stroke $\cdot$ Ischemic infarction

\begin{abstract}
Endovascular technology was initially applied in treating peripheral vascular disease and was further developed in managing coronary artery disease. During the latest two decades, it has been introduced into the arena of cerebrovascular diseases, which has garnered attention and research interests.

Xinfeng Liu

Department of Neurology, Jinling Hospital

Nanjing University School of Medicine

305 East Zhongshan Road

Nanjing 210002, Jiangsu Province (PR China)

E-Mail xfliu2@vip.163.com
Suming Zhang

Department of Neurology, Tongji Hospital, Tongji Medical College Huazhong University of Science and Technology 1095 Jiefang Avenue, Wuhan 430030 (PR China)

E-Mail summing_zhang@163.com 
The technology roadmap and treatment strategy for patients with ischemic stroke vary widely due to the different professional background of the interventional practitioners. Thus, it was crucial to set up a series of guidelines in order to conduct standardized clinical practice. Based on this consideration, the American Heart Association (AHA), the European Stroke Organization (ESO), the American Academy of Neurology (AAN) and the Society of Interventional Radiology (SIR) individually released their guidelines for the interventional treatment of cerebrocervical vascular diseases in recent years according to extensive previous clinical researches. Clinical factors ranging from patient selection, peri-/postoperative monitoring for prevention and the treatment of complications were ordered in these guidelines. The interventional approach for treating carotid stenosis was involved in the Chinese Guidelines for Secondary Prevention of Ischemic Stroke and Transient Ischemic Attack 2010, but no detailed recommendations were provided. As a result of sharply increased numbers of candidates for endovascular treatment and rapidly improved interventional skills and equipment, specialized guidelines in this field were needed urgently.

The writing committee limited the scope of this document to the interventional field of comprehensive diagnosis and treatment of ischemic stroke. We compiled the manuscript with reference to other important guidelines around the world as well as reviewing the latest evidence-based results. The recommendations of the guideline are intended to assist clinicians in standardized evaluation and to stratify the treatment of patients who have suffered an ischemic stroke due to artery stenosis or occlusion.

\section{Catheter-Based Contrast Angiography}

In recent years, followed by the rapid progress of angiography, it was more convenient to obtain carotid and cerebrovascular imaging noninvasively by means of vascular ultrasound, CT angiography (CTA) and magnetic resonance angiography (MRA). As a result, the clinical utility of conventional catheter-based digital subtraction angiography (DSA) became significantly limited. DSA has an approximately $0.5 \%$ risk for major complications, and the cost is considerable due to hospitalization and dispensable catheters [1]. However, under special conditions, it might be essential to know the location and severity of the vascular lesion comprehensively and dynamically, in order for clinicians to be able to make specific treatment decisions regarding a surgical/interventional procedure. Under such circumstances, DSA would be irreplaceable as the gold standard of angiography.

\section{Indications and Contraindications for DSA}

Due to the invasive nature of DSA, it is advisable to demonstrate the indications and contraindications. In principle, minor or noninvasive examination in the form of carotid ultrasound, transcranial Doppler ultrasound (TCD), MRA or CTA should be conducted prior to DSA in patients with ischemic stroke. DSA should only be considered if noninvasive angiography were unattainable or unable to provide sufficient information for diagnosis or treatment. There are also cases where a patient suspected of having had an acute cerebral infarction or subarachnoid hemorrhage that needs emergent interventional procedure may be considered as a DSA candidate [2].

It is of note that the indications and contraindications of DSA listed below are only general principles. Specific decisions regarding individuals should be based on comprehensive assessment and careful consideration of the clinical status of the patients [3]. 
Suggested Indications for DSA

(1) The purpose of DSA includes examining the vascular etiology of ischemic stroke and other CNS diseases (it includes the arteries, veins and capillaries).

(2) Looking for causes of intracranial hematoma or subarachnoid hemorrhage.

(3) Preoperative examination of blood-rich tumor in the head and face.

(4) To identify the kind of tumor or determine the relationship between the blood supply to the arteries and intracranial space-occupying lesions.

(5) To determine the pathological changes of cerebrocervical arteries and the anatomical relationship before interventional or surgical treatment.

(6) To determine the accurate location and severity of cerebrovascular disease before intra-artery thrombolysis.

(7) To evaluate face/head or intracranial vascular disease during follow-up periods.

Contraindications for DSA

(1) Allergy to iodine or contrast agent.

(2) Allergy to metal or radiography equipment.

(3) Severe hemorrhagic disease or hemorrhagic tendency (e.g. platelet count $\leq 80 \times 10^{9} / 1$ ).

(4) Severe cardiac/liver/kidney insufficiency or creatinine (Cr) $\geq 250 \mu \mathrm{mol} / \mathrm{l}$.

(5) Uncontrolled generalized infection or local infection located at the access site

(6) Cerebral hernia or other life-threatening situations.

\section{Preoperative Preparation}

Regular physical inspections 1 day before DSA on interventional-treatment candidates were necessary to determine contraindications to the procedure. (1) A patient with $\mathrm{Cr} \leq 250$ $\mu \mathrm{mol} / \mathrm{l}$ was usually safe for a DSA procedure with a limited dose of contrast agent $(<100 \mathrm{ml})$. (2) Patients with platelet count $\leq 80 \times 10^{9} / \mathrm{l}$ should not be recommended for DSA. (3) Patients who have been on anticoagulation treatment need to replace warfarin with heparin days before the procedure until the international normalized ratio (INR) decreases below 1.4. (4) Patients with grade II or III cardiac function should be treated carefully during DSA with a low dose of contrast agent and a short procedure time. (5) Before the DSA procedure, it is important to inform patients and their families of the necessity and potential risk. The proportion of procedurerelated complications (including stroke and death) is $0.3 \%$ in asymptomatic patients and $0.5 \%$ in symptomatic patients, respectively. DSA could lead to disastrous results in some cases despite the extremely low complication rates. As a result, clinicians should not overemphasize the safety or danger of DSA. (6) Written informed consent should be obtained from all patients.

\section{Pre-/Intraoperative Medication}

Venous canals should be established before the DSA procedure for potential untoward reactions or complications. Corresponding treatment should be immediately needed in case of contrast-agent allergy, vasospasm, hypotension or bradycardia. A sedative in the form of 0.1-0.2 g phenobarbital by intramuscular injection before or during the procedure or a perioperative diazepam/midazolam intravenous (i.v.) infusion should be considered in patients who have a dread of the coming procedure. Patients should be under electrocardiogram and vital-signs monitoring for the whole procedure. At the same time, partial heparinization (Liquaemin 2,000 U i.v. bolus and heparin-saline infusion via catheter) is also mandatory for patients weighing $60-80 \mathrm{~kg}$.

\section{Complications and Treatment}

The major complications of DSA should be controlled under $0.3-0.5 \%$ in experienced institutions. The most frequent complications and the corresponding treatments are listed below. 
Cerebral Vasospasm

Most cerebral vasospasm during the DSA procedure was attributed to the stimulation of the catheter or guide-wire rather than to the contrast agent. Generally, the consequences were not serious unless the vasospasm was long enough to cause a cerebral ischemic event. In such a case, anticonvulsants such as papaverine or nitroglycerine should be infused intra-arterially via a catheter. Cessation of the stimulation is definitely the most effective method against vasospasm.

Ischemic Stroke

Symptomatic or asymptomatic perioperative ischemic stroke was mostly caused by cerebral embolism from the residue of vascular plaques or thrombosis attached to the catheter; it could, to a small degree, have come from air embolism due to improper manipulation during the procedure. The prevention methods include: (1) systematic heparinization before the procedure, which could effectively reduce thrombosis overlapping the interventional catheter, (2) an angiogram beginning with the aortic arch, going to the supra-arch major artery, and then a secondary and super-selective branch angiogram; the guide-wire and catheter should avoid passing through vulnerable plaques overlapping the vascular wall, and (3) air embolism should be strictly guarded. It is usually difficult to distinguish thrombopoiesis from thrombolysis. The former could be treated by thrombolysis, but no effective treatment was available for thrombosis. Air embolism could be immediately treated with hyperbaric oxygenation and the outcome is usually favorable.

Groin Hematoma and Pseudoaneurysm

Groin hematoma and pseudoaneurysm were usually caused by improper compressing after the procedure or repeated femoral artery puncture, which could lead to injury of the femoral artery branches. Furthermore, insufficient compressing time of the access site or untimely load-bearing of the involved leg could also be reasons for groin hematoma and pseudoaneurysm.

\section{Retroperitoneal Hematoma}

Retroperitoneal hematoma was usually due to excessive artery accessing and improper manipulation of the guide-wire, which led to penetration of the posterior wall of the iliac artery. No sufficient pressure could be applied to stop the blood flow because of a lack of tough and tensile tissue. Surgery should be considered immediately if the guide-wire or catheters damage the vascular wall of the iliac artery, especially when severe atherosclerosis or aneurysm is located.

Femoral or Iliac Artery Dissection

Femoral artery or iliac artery dissection was largely caused by the subintimal injury due to improper manipulation of the needle or catheter without the opportune awareness of the operators. As a result of the intima crevasse located distal to the dissection, the dissection would not extend without continuous stimulation of the catheter and will heal itself in a few hours or days after injury. However, if the dissection is deep or there are indications for unstable hemodynamics, local angiography and surgical consultation should be considered.

Vagal Reflex

Vagal reflex usually occurred when the sheath was removed outwards. A decline in heart rate and blood pressure, paleness or a cold sweat were typical presentations of vagal reflex. In patients of advanced age and defective cardiac function, it may lead to a life-threatening prognosis. Atropine by i.v. injection is the primary choice, while at the same time blood volume should be sufficiently replenished. 
Liu et al.: Chinese Guidelines for Endovascular Management of Ischemic

Cerebrovascular Diseases

\section{Cortical Blindness}

A few cases of perioperative cortical blindness were reported in the literature. All patients were fully recovered a few hours or days after the procedure. The specific mechanism remains undetected. It was speculated that this was due to the excessive concentration and dosage of contrast agent or the catheter-based cerebral vasospasm. There is no effective treatment for cortical blindness. Appropriate rehydration and vascular antispasmodic drugs may be considered.

\section{Recommendation}

Patients should be screened before the DSA procedure, and necessity and risks must be comprehensively evaluated. Noninvasive inspection could be performed prior to DSA if necessary (Level I).

\section{Thrombolytic Therapy for Acute Ischemic Stroke}

As brain tissue is more vulnerable to ischemic injury than other tissues, inconvertible damage could be inevitable shortly after occlusion of the cerebral artery. Thus, treatment should be performed within an effective time window in order to save neurons on the verge of necrosis.

Emergent interventional therapies based on this theory are contact intra-arterial thrombolysis, intra-arterial mechanical embolectomy surgery, intra-artery mechanical thrombectomy surgery and ultrasound-assisted thrombolysis. For patients suffering ischemic stroke, the key point is to carry out treatment as soon as possible. How to recognize stroke patients, how to conduct a rapid inspection and how to select the most rational treatment option turned out to be the most important parts of the comprehensive treatment.

\section{Latest Evidence of Interventional Treatment on Acute Ischemic Stroke}

In multiple randomized controlled trials, it has been confirmed that thrombosis with recombinant tissue plasminogen activator (r-TPA) is beneficial for patients with acute ischemic stroke (0-4.5 h) [4-7]. However, other studies indicate that the proportion of i.v. recanalization was unsatisfactory, especially when the lesion was located in major arteries (e.g. the carotid artery) or the forking of the arteries (e.g. carotid bifurcation). Furthermore, it was difficult to completely avoid reocclusion. Therefore, it is still necessary to investigate other treatment options for improving the clinical prognosis. Percutaneous catheter-based intra-arterial thrombosis has therefore turned to be one of the hot-spots in recent years.

Theoretically, the contact surface between thrombolytic medication and atherosclerotic plaques increases during the procedure. Local concentration of thrombolytic medication would also elevate. Combined with direct mechanical thrombectomy and embolectomy, the proportion of recanalization of the occluded vessel would therefore be significantly improved. On the other hand, the intra-arterial thrombolysis is generally carried out under fluoroscopy, so whether occlusive vessels have been recanalized could be observed directly from the angiogram. However, the prerequisite DSA performance before intra-arterial thrombolysis would prolong the 'time to needle'. The clinical effect would therefore be doubted when compared with i.v. thrombolysis. Moreover, the strict requirements regarding operators and equipment also limited the utility in clinical practice.

Prolyse in Acute Cerebral Thromboembolism (PROACT) was a clinical randomized, double-blind controlled trial that included patients with middle cerebral artery occlusion within $6 \mathrm{~h}$ [8]. The safety and efficacy of intra-arterial thrombolysis were evaluated by comparison to patients who were treated with $6 \mathrm{mg}$ recombinant prourokinase and placebo 
by catheter-based localized thrombolysis. The results indicated a recanalization rate of $58 \%$ in the treatment group and $14 \%$ in the placebo group $(p=0.017)$. Prognosis significantly favored the recombinant prourokinase group, while the incidence of symptomatic hemorrhage did not differ in the two arms (15 vs. 14\%). Moreover, the National Institute of Neurological Disorders and Stroke (NINDS) registry study also confirmed the advantages of intraarterial thrombolysis in a specific group of patients [9].

In order to improve the recanalization rate of patients with major artery occlusion, some new techniques like direct mechanical balloon dilation, mechanical embolectomy, capture device, laser/ultrasound-assisted thrombolysis and intra-arterial/venous glycoprotein IIb/ IIIa inhibitor infusion have been adopted in clinical practice. However, there are still only limited clinical trials on these treatments.

Recently, a new embolectomy device was employed in the Mechanical Embolus Removal in Cerebral Ischemia (MERCI) trials which recanalized the occlusive vessels with a proportion of $45 \%$ [10]. This has been confirmed to be a reasonable approach for treating selected patients with ischemic stroke. However, further investigation is needed to determine whether this and other embolectomy devices will indeed improve the long-term prognosis of patients.

\section{Indications and Contraindications for Intra-Arterial Thrombolysis} Indications

(1) Age 18-80 years.

(2) Ischemic infarction evolved in anterior circulation within $6 \mathrm{~h}$, posterior circulation territory ischemic stroke within $24 \mathrm{~h}$.

(3) NIHSS score: 4-24.

(4) Intracerebral hemorrhage and irreversible damage of brain tissue should be excluded by CT or other examination.

(5) Written informed consent should be obtained from all patients.

Relative Contraindications

(1) Previous intracranial hemorrhage including suspicious subarachnoid hemorrhage.

(2) Head trauma within 3 weeks.

(3) Major surgical operations within 2 weeks.

(4) Arterioaccess located where compression hemostasis would be difficult.

(5) Cerebral infarction or myocardial infarction within 3 months (obsolete lacunar infarction without neurological deficit not included).

(6) Severe heart, liver and kidney dysfunction or severe diabetes.

(7) Active bleeding or trauma found by physical examination.

(8) On anticoagulation therapy with an INR $>1.5$.

(9) Previous heparin therapy within $48 \mathrm{~h}$ with a higher value of activated partial thromboplastin time.

(10) Platelet count $\leq 80 \times 10^{9} / \mathrm{l}$.

(11) Blood glucose $<2.7 \mathrm{mmol} / \mathrm{l}$.

(12) Systolic pressure $>180 \mathrm{~mm} \mathrm{Hg}$ or diastolic pressure $>100 \mathrm{~mm} \mathrm{Hg}$.

(13) Pregnancy.

(14) Rapidly healed clinical symptoms.

(15) Improper cooperation of patients.

Perioperative Management and Treatment of Complications of Intra-Arterial Thrombolysis The dose of r-TPA in intra-arterial thrombolysis is usually $1 / 3$ of the i.v. dose. No more than $22 \mathrm{mg}$ was used in most of the studies. Proximal to occlusion, 1-2 mg r-TPA was injected 
via catheter. Infusion of the rest of the r-TPA distal of the occlusion was performed after the microcatheter went over the occlusive segment of vessels with a speed of $1 \mathrm{mg} / \mathrm{min}$ or impulsive injection. Urokinase thrombolysis could also be adopted in intra-arterial thrombolysis with a limited dose of 600,000 $\mathrm{U}$.

Hemorrhage is the most common complication in thrombolytic therapy, occurring intracranially or in other organs. A blood test, in particular a coagulation test, should be carried out at once if a hemorrhage is suspected. Emergent CT scan was reasonable in patients with suspicious intracranial hematoma. If hemorrhage is confirmed, treatment according to the Guidelines for Cerebral Hemorrhage Treatment in China 2010 [11] should be performed as soon as possible, with a neurosurgical consultation and even surgery being considered if necessary.

All patients should be under comprehensive surveillance in ICU right after the procedure, regardless of the incidence of revascularization or not. Vital signs and neurological signs should also be carefully individually monitored every 15 and $30 \mathrm{~min}$ in the first $3 \mathrm{~h}$. Antiplatelet therapy was not suggested in the first $24 \mathrm{~h}$ after the intra-arterial thrombolysis procedure except for in the patients who only underwent mechanically-assisted embolectomy.

Recommendations

(1) Intra-arterial thrombolysis should be performed in institutions qualified for catheterbased angiography and interventional treatment (Level I, Evidence C).

(2) Intra-arterial thrombolysis is an alternative for treating patients with contraindications to i.v. thrombolysis (Level II, Evidence C).

(3) Intra-arterial thrombolysis could be applied in selected patients with acute cerebral infarction due to middle cerebral artery occlusion within $6 \mathrm{~h}$ (Level II, Evidence B).

(4) For patients with severe cerebral infarction involved in posterior circulation within 6-24 h, intra-arterial thrombolysis could be performed through strict evaluation and selection (Level III, Evidence C).

(5) r-TPA and urokinase infusion were both qualified for intra-arterial thrombolysis (Level I, Evidence C).

\section{Interventional Treatment for Carotid Stenosis}

According to previous research, approximately $25 \%$ of strokes can be attributed to carotid stenosis or occlusion. Multiple randomized clinical trials confirm that carotid endarterectomy (CEA) could reduce the risk for ischemic stroke in patients with moderate-tosevere symptomatic $(>50 \%)$ and asymptomatic $(60 \%)$ carotid stenosis. In recent years, followed by the rapid progression of catheter-based techniques and the application of a cerebral protection device, carotid artery stenting (CAS) has become an alternative for the treatment of CEA in selected patients. This section mainly focuses on atherosclerotic stenosis; radiation-induced stenosis and carotid dissection were also involved.

\section{Evaluation of Atherosclerotic Stenosis}

Symptoms Evaluation

Comprehensive physical examination, particularly neurological examination, includes heart carotid bruit auscultation as well as retinal thrombosis speculation via ophthalmoscopy which are required in patients with suspicious atherosclerotic carotid stenosis. The NIHSS score can be employed to evaluate the neurological functional deficits. Clinical presentation and positive signs must echo the neurovascular imaging in order to identify whether ipsilateral atherosclerotic carotid stenosis contributes to the intracranial lesion and symptoms. This is the key procedure for distinguishing symptomatic from asymptomatic carotid stenosis/occlusion. 
Imaging Evaluation

Carotid ultrasound, MRA and CTA were widely used in the primary assessment in most patients with carotid stenosis. Both the North American Symptomatic Carotid Endarterectomy Trial (NASCET) and the European Carotid Surgery Trial (ECST) utilized invasive angiography as the primary method to assess the severity of carotid stenosis. DSA is now irreplaceable in China, despite the fact that noninvasive methods like carotid ultrasound or CTA could also be helpful in stenosis speculation before intravascular procedures. Carotid stenosis is regularly calculated according to the NASCET.

\section{Medical Treatment of Atherosclerotic Stenosis}

Refer to the Chinese Guidelines for Secondary Prevention in Patients with Ischemic Stroke and Transient Ischemic Attack 2010 [12].

\section{Evidence of Carotid Angioplasty and Stenting}

Prevention of ischemic stroke is the primary aim of carotid revascularization. For the most part, ischemic stroke caused by carotid stenosis can be attributed to embolism and thrombosis; to a lesser degree, stroke is caused by hypoperfusion. As a result, treatment for carotid atherosclerotic stenosis should mainly focus on how to prevent the thrombus from falling down and mitigate the hemodynamic compromise. Treatment with drugs, surgery and endovascular interventional treatment are the three current options for patients with carotid atherosclerotic disease.

The Stenting and Angioplasty with Protection in Patients at High Risk for Endarterectomy (SAPPHIRE) trial [13] enrolled 334 patients with symptomatic carotid stenosis of at least $50 \%$ of the luminal diameter or asymptomatic carotid stenosis of more than $80 \%$. All patients had one or more high-risk factors for CEA (radiation vascular stenosis, pro-CEA restenosis, a history of myocardial infarction or stenosis in a high/low position which makes CEA difficult). The result of the study showed that the proportion of perioperative myocardial infarction, stroke and death within 30 days in the CAS and CEA groups was 4.8 and $9.8 \%$, respectively $(\mathrm{p}=0.01)$. The primary end point was observed in $12.2 \%$ of the CAS group and in $20.1 \%$ of the CEA arm. The study demonstrated that CAS was superior to CEA in treating patients with a high risk of surgical therapy according to the noninferiority test.

Another 2 multicenter randomized contrast clinical trials, the Stent-Protected Angioplasty versus Carotid Endarterectomy (SPACE) trial and the Endarterectomy Versus Angioplasty in Patients with Symptomatic Severe Carotid Stenosis (EVA-3S) trial, took CEA as the gold standard to evaluate the feasibility of CAS by noninferiority test [14, 15]. They both confirmed that CAS was as effective as CEA in treating atherosclerotic carotid stenosis. The Carotid Revascularization Endarterectomy versus Stenting Trial (CREST) employed a more strict qualification standard for operators; the primary end point included stroke, myocardial infarction, perioperative death for all reasons and ischemic stroke ipsilateral to the 'criminal artery' within 4 years [16]. The primary end point in the CAS group was $7.2 \%$ versus $6.8 \%$ in CEA group without a significant difference $(\mathrm{p}=0.51)$.

It should be noted that in the USA and Europe, CEA has been performed by skilled doctors for over 50 years. However, in some developing countries such as China, only a limited number of institutions and doctors received standard fellowship of CEA. On the other hand, relatively more doctors paid attention to CAS and completed sufficient training, so CAS as an alternative toCEA could play a more important role in China than in Western countries [17].

For carotid dissection, there is no randomized controlled trial to date. The clinical data were derived from a limited number of cohort studies. 
Liu et al: Chinese Guidelines for Endovascular Management of Isch

Cerebrovascular Diseases

\section{Perioperative Management and Prevention of Complications}

Antiplatelet medication such as aspirin (100-300 mg) and clopidogrel (75-300 mg), at least, should be administrated to patients $24 \mathrm{~h}$ before CAS. Sedatives should be considered based on the specific condition of the patient. Intraoperative monitoring of oxygen saturation and vital signs should also be mandatory throughout the procedure as well as anticoagulation (heparin $70 \mathrm{U} / \mathrm{kg}$ ). A cerebral protection device was also recommended in recent studies [18].

After the procedure, there should be regular monitoring of the access site and vital/ neurological signs. Lifelong administration of aspirin and clopidogrel for at least 1 month is reasonable if there are no contraindications.

The perioperative complications of CAS include cerebral embolism, thrombosis, intracranial hemorrhage and hyperperfusion syndrome. Interventional manipulation should stop immediately once focal neurofunctional compromise is confirmed during the procedure. Look for the specific cause such as vasospasm, vascular occlusion or intracranial hemorrhage by DSA and then actively deal with it. Thrombolysis or embolectomy therapy could be performed to solve acute occlusion of major arteries. Intracranial hemorrhage and hyperperfusion syndrome could occur within several days or weeks after the procedure. Improper management of blood pressure and bilateral severe stenosis of the major arteries before the procedure could increase the risk of hyperperfusion syndrome; if this occurs, strict control of blood pressure and intracranial pressure plus symptomatic treatment should be applied at once.

Indications and Contraindications of CAS

Indications

(1) $\geq 18$ years old

(2) Symptomatic carotid stenosis $\geq 50 \%$, asymptomatic stenosis $\geq 70 \%$.

(3) Informed consent should be obtained before the procedure.

Relative Contraindications

(1) Intracranial hemorrhage within 3 months.

(2) Cerebral aneurysm which was not able to be treated pre-/intraprocedure.

(3) Myocardial infarction or major cerebral infarction within 2 weeks.

(4) Gastrointestinal disease with active bleeding.

(5) Uncontrolled hypertension.

(6) Heparin, aspirin or other antiplatelet treatment contraindicated.

(7) Allergic to contrast agent or materials or equipment of the catheter-based angiography.

(8) Severe heart, liver, kidney or lung disease.

(9) Tortuous or variant vasculature, difficult for a delivery system or stent to go through.

(10) Wide-ranging plaques or stenosis.

(11) Inflammatory vascular injury or widely distributed abnormal artery construction.

(12) Thrombosis and severe calcification inside the atherosclerotic lesions.

(13) Uncontrolled systemic infection or local infection near the access site.

(14) A state of unconsciousness or severe neurofunctional compromise.

\section{Recommendations}

(1) For symptomatic patients with carotid stenosis of more than 50\%, CAS should be performed as an alternative to CEA (Level I, Evidence B).

(2) CEA and CAS should be performed at least 2 weeks after major cerebral infarction. In cases without contraindications, they should be considered within 2 weeks (Level I, Evidence B). 
(3) For asymptomatic patients with carotid stenosis of more than $70 \%$, CAS should be indicated as an alternative to CEA (Level I, Evidence B).

(4) Aspirin combined with clopidogrel should be administrated daily before the procedure and at least 1 month after the procedure (Level I, Evidence C).

(5) For other secondary prevention measures, refer to the Chinese Guidelines for Secondary Prevention in Patients with Ischemic Stroke and Transient Ischemic Attack 2010.

(6) CAS should be performed in institutions with a proportion of perioperative disability and death $<6 \%$ (Level I, Evidence B).

\section{Interventional Treatment of Extracranial Vertebral Arteries}

Approximately $25 \%$ of ischemic stroke occurs within the territory of the vertebral-basilar artery. The most important etiology has proved to be atherosclerotic stenosis. The clinical symptoms in considerable numbers of patients with vertebral-basilar artery stenosis will never be significantly alleviated despite best medical treatment. Considering the wide application of percutaneous transluminal coronary angioplasty in coronary arteries, vertebral angioplasty and stenting is therefore now becoming a hot-spot in the arena of interventional neurology.

\section{Evaluation of Vertebral Artery Stenosis}

The most regularly used methods in vertebral artery examination include vertebral artery ultrasound, TCD, MRA, CTA and DSA. Ultrasound is especially helpful in detecting atherosclerotic plaques overlapping the artery wall, while TCD has the advantage of hemodynamic assessment and microemboli detection. Compared to DSA, the sensitivity and specificity of MRA in the assessment of vertebral artery stenosis is $70-100 \%$. The disadvantage of MRA is that the description of distal vessels is not regularly sufficient for clinical requirement.

A diagnostic DSA procedure is especially helpful to identify the position, length, diameters, eccentricity and perforating branches of the involved arteries and adjacent vessels. In addition, whether the posterior communicating artery is open and the external carotid artery-vertebral artery collateral bypass exists can be clearly and dynamically displayed by catheter-based angiography. However, being an invasive procedure, $0.5 \%$ of complications are inevitable.

\section{Evidence of Vertebral Artery Angioplasty and Stenting}

The Carotid and Vertebral Artery Transluminal Angioplasty Study (CAVATAS) was a prospective, multicenter randomized controlled trial [19]. One of the subtype analyses randomized 16 patients with vertebral artery stenosis into an endovascular-treatment group and a best-medicine treatment group. The endovascular procedure succeeded in $100 \%$ of the patients, 2 of them then underwent perioperative TIA and no death or stroke within the territory of the artery involved were observed up to 30 days after the procedure. In the 4.7 years of follow-up, no primary end point was defined as vertebral-basilar artery stroke in both groups. However, 3 patients of each group died of myocardial infarction or stroke within the carotid artery territory. The study demonstrated that patients with vertebral artery stenosis are more likely to get myocardial infarction and carotid stroke than stroke within the posterior circulation. Interventional therapy has not yet been proven superior to medical treatment.

\section{Perioperative Management of Interventional Treatment of Atherosclerotic Vertebral Artery Stenosis}

In patients with bilateral vertebral stenosis, the symptomatic or dominant side should be given priority. There is no evidence on interventional treatment of the chronic vertebral artery occlusion to date. As a result, the utility of this therapy is limited to the field of clinical 
trials. Aspirin (100-325 mg/day) and clopidogrel (75 mg/day) should be administrated 3-5 days before endovascular treatment of the vertebral artery. For the emergent procedure, a loading dose of antiplatelet drugs (aspirin and clopidogrel, $300 \mathrm{mg}$ each) should be mandatory. Atherosclerotic vertebral artery disease usually involves the subclavian artery; the proximal stent should therefore extend $2 \mathrm{~mm}$ into the subclavian artery. The proportion of restenosis would increase if the stent covers only the edge of the vertebral artery or fails to cover the lesion completely. In contrast, if the stent extends too much into the subclavian artery, erythrocytes could be mechanically destroyed. After the procedure, clopidogrel should be administered regularly for at least 1 month and aspirin for life. The treatment for perioperative complications is similar to that of CAS.

The indications and contraindications for vertebral artery stenting are similar to that of CAS.

Recommendations

(1) For symptomatic patients with more than $50 \%$ vertebral artery stenosis, angioplasty and stenting should be considered in the case of no response to medical treatment (Level II, Evidence C).

(2) For asymptomatic patients with more than $70 \%$ vertebral artery stenosis, angioplasty and stenting should be considered in the case of progressive atherosclerotic lesions (Level II, Evidence C).

(3) For asymptomatic patients with severe vertebral artery stenosis (more than 70\%), angioplasty and stenting should be considered in the case of congenital dysplasia or absence of the contralateral vertebral artery (Level II, Evidence C).

(4) For symptomatic patients with more than 50\% subclavian artery stenosis, angioplasty and stenting should be considered in the case of no response to medical treatment (Level II, Evidence C).

(5) For patients with vertebral/subclavian artery stenosis, aspirin combined with clopidogrel should be administered before the procedure and for at least 1 month afterwards (Level III).

(6) Vertebral/subclavian artery angioplasty and stenting should be performed in institutions with low perioperative complications (Level III).

\section{Interventional Treatment of Intracranial Arteries}

Atherosclerotic intracranial artery stenosis is one of the most important causes of ischemic stroke worldwide. It has been demonstrated that $8-10 \%$ of ischemic strokes are caused by intracranial atherosclerotic disease. However, in Chinese patients, more than $30 \%$ of ischemic stroke is due to intracranial artery disease. Medical treatment of intracranial lesions is not meeting the clinical requirements sufficiently. The safety and effectiveness of interventional therapy of intracranial stenosis also remains unclear.

\section{Clinical Evaluation of Intracranial Atherosclerotic Stenosis}

Candidates for intracranial angioplasty and stenting should receive comprehensive clinical evaluation of neurofunction status and risk factors for cerebrovascular disease, which include the traditional ones such as age, gender, smoking, hypertension, diabetes, dyslipidemia as well as recently mentioned ones such as C-reactive protein or plasma homocysteine levels. The assessment of heart, lung and kidney functions is also important for a smooth endovascular procedure. Patients should be surveyed by scales like the Barthel Index, modified Ranking Scales and the NIHSS in order to quantify the neurofunction status before and after the procedure. Moreover, the clinical symptoms and signs should be evaluated to 
assess the association of the involved arteries and to exclude other diseases with the potential for being treated in a noninterventional way, such as moyamoya disease or vasculitis. The physicians in charge of these tasks should receive sufficient specialized training.

\section{Imaging Evaluation of Intracranial Atherosclerotic Stenosis}

(1) Intracranial vascular evaluation: contrast-enhanced MRA, CTA or DSA should be performed in patients with intracranial artery stenosis or occlusion. DSA and CTA are more accurate than MRA in evaluating stenosis, according to a previous contrast study. Thus, to evaluate arteries within the Willis Circle, DSA or CTA is suggested prior to MRA. DSA is suggested for the evaluation of arteries outside the Circle of Willis.

(2) Calculation of stenosis of atherosclerotic intracranial artery stenosis: it is critical to accurately measure the original diameter of the vessels and the extent of atherosclerotic lesion because the selection of stenting and balloon mostly depend on this. The length and diameters of the balloon are also based on the extent and severity of the stenosis and the diameters of the normal arteries adjacent to the lesions. As a result of the inherent anatomical difference between extracranial and intracranial arteries (the latter are more tortuous, more slender and giving out more branches) the methods for the evaluation of extracranial arteries are not appropriate for intracranial arteries. The Warfarin Aspirin Symptomatic Intracranial Disease (WASID) trials built up a series of reliable methods to evaluate the severity of intracranial artery stenosis [20].

(3) Evaluation of range and severity of atherosclerotic intracranial artery stenosis: Mori et al. [21] suggested a classification based on the angiographic character for predicting the clinical prognosis of intracranial angioplasty. According to the length and geometric morphometrics of atherosclerotic lesions, the patients were divided into 3 categories: type A indicated a short $(\leq 5 \mathrm{~mm})$, concentric or moderate eccentric lesion, type B indicated a tubular (range 5-10 mm), extremely eccentric and moderate angulated lesion and type $C$ indicated a diffused $(>10 \mathrm{~mm})$ or proximal part of extremely angulated tortuous lesions. The more complex the lesion, the worse the short-/long-term prognosis will be. The Mori classification now is widely used in the description of intracranial stenting candidates, although it was first suggested for the field of angioplasty.

\section{Medical Treatment of Atherosclerotic Intracranial Artery Stenosis}

Refer to Chinese Guidelines for Secondary Prevention in Patients with Ischemic Stroke and Transient Ischemic Attack 2010 [12].

\section{Evidence of Intracranial Vertebral Artery Angioplasty and Stenting}

Stenting of symptomatic atherosclerotic lesions in the vertebral or intracranial arteries (SSLVIA) was a multicenter prospective clinical trial performed to evaluate stenting in treating intracranial artery stenosis [22]. Sixty-one patients including 43 patients with symptomatic intracranial stenosis and 18 patients with extracranial vertebral stenosis were prospectively enrolled in this study. The technical success was 95\%. Within 30 days of the procedure, $7.2 \%$ patients had suffered strokes and no patients had died. The proportion of restenosis in 6 months was 35\% and stroke involving the treated vascular territory was $10.9 \%$.

The Wingspan trial was another prospective multicenter clinical trial focussing on 45 patients with intracranial stenosis (50-99\%) who had recurrent stroke despite best medical treatment [23]. The proportion of technical success was $97.7 \%$. The proportion of strokes and death within 30 days of the procedure was $4.5 \%$. In the long-term follow-up, ipsilateral stroke occurred in $9.3 \%$ of patients in 1 year. Restenosis in 6 months occurred in $9.3 \%$ of patients, but no one suffered ischemic stroke. Numbers from a previous single-center observational study suggest the moderate feasibility and safety of intracranial stenting [24]. 
Liu et al.: Chinese Guidelines for Endovascular Management of Ischemic

Cerebrovascular Diseases

Perioperative Management of Interventional Treatment of Atherosclerotic Intracranial Artery Stenosis

The combination of aspirin and clopidogrel should be administrated to candidates for intracranial artery stenting to reduce perioperative platelet-related embolus. Agreement on the safety, specific dosage and combinations of antiplatelet has not yet been reached. So far, the most widely used proposal is that $100-300 \mathrm{mg}$ aspirin should be administered per day if the patients are on long-term aspirin treatment, while $300 \mathrm{mg}$ aspirin should be mandatory at least $2 \mathrm{~h}$ ( $24 \mathrm{~h}$ is recommended) before the procedure in patients not receiving regular antiplatelet treatment. After the procedure, dual antiplatelet and hyperbaric oxygenation therapy is recommended, clopidogrel should be added for at least 1 month, and aspirin for life.

Intracranial hematoma and ischemic events are the most common and most severe complications of intracranial interventional manipulation. The former is the most important cause of intraoperative death, presenting as sudden-onset severe headache, nausea, vomiting and rapidly declined consciousness. An emergent CT scan as soon as possible is suggested in the case of suspicion of intracranial hematoma. The medical treatment of hematoma should focus on managing the blood pressure and reducing or controlling subsequent bleeding. Infusion of platelets, protamine or fresh frozen plasma would also be reasonable if necessary. Neurosurgical intervention should be considered in the case of a large amount of bleeding. In patients with transient or permanent new-onset perioperative neurological signs, the evaluation of treatment arteries and other vessels would be required because perioperative ischemic stroke could be caused by acute thrombosis in a stent or by vasospasm. If acute thrombosis is confirmed, emergent thrombolysis should be considered alongside antithrombotic medicine.

\section{Recommendations}

(1) For patients with symptomatic intracranial stenosis, medical treatment is recommended in the first instance (Level I, Evidence A). Refer to the Chinese Guidelines for Secondary Prevention in Patients with Ischemic Stroke and Transient Ischemic Attack 2010 in detail. Intracranial angioplasty and stenting in qualified institutions are advisable in the case of no response to medical treatment (Level III, Evidence C).

(2) For patients with asymptomatic intracranial stenosis, intracranial angioplasty and stenting are not recommended (Level I, Evidence A).

\section{References}

- 1 Pryor JC, Setton A, Nelson PK, Berenstein A: Complications of diagnostic cerebral angiography and tips on avoidance. Neuroimaging Clin N Am 1996;6:751-758.

2 Willinsky RA, Taylor SM, TerBrugge K, Farb RI, Tomlinson G, Montanera W: Neurologic complications of cerebral angiography: prospective analysis of 2,899 procedures and review of the literature. Radiology 2003; 227:522-528.

3 Barr JD: Cerebral angiography in the assessment of acute cerebral ischemia: guidelines and recommendations. J Vasc Interv Radiol 2004;15:S57-S66.

- 4 Hacke W, Kaste M, Bluhmki E, Brozman M, Dávalos A, Guidetti D, Larrue V, Lees KR, Medeghri Z, Machnig T, Schneider D, von Kummer R, Wahlgren N, Toni D, ECASS Investigators: Thrombolysis with alteplase 3 to $4.5 \mathrm{~h}$ after acute ischemic stroke. N Engl J Med 2008;359:1317-1329.

- 5 The National Institute of Neurological Disorders and Stroke rt-PA Stroke Study Group: Tissue plasminogen activator for acute ischemic stroke. N Engl J Med 1995;333:1581-1587.

6 Hacke W, Kaste M, Fieschi C, Toni D, Lesaffre E, von Kummer R, Boysen G, Bluhmki E, Höxter G, Mahagne MH, Hennerichi M: Intravenous thrombolysis with recombinant tissue plasminogen activator for acute hemispheric stroke: the European Cooperative Acute Stroke Study (ECASS). JAMA 1995;274:1017-1025. 
- 7 Hacke W, Kaste M, Fieschi C, von Kummer R, Davalos A, Meier D, Larrue V, Bluhmki E, Davis S, Donnan G, Schneider D, Diez-Tejedor E, Trouillas P, Second European-Australasian Acute Stroke Study Investigators: Randomised double-blind placebo-controlled trial of thrombolytic therapy with intravenous alteplase in acute ischaemic stroke (ECASS II). Lancet 1998;352:1245-1251.

- 8 Furlan A, Higashida R, Wechsler L, Gent M, Rowley H, Kase C, Pessin M, Ahuja A, Callahan F, Clark WM, Silver F, Rivera F: Intra-arterial prourokinase for acute ischemic stroke. The PROACT II study: a randomized controlled trial. Prolyse in Acute Cerebral Thromboembolism. JAMA 1999;282:2003-2011.

- 9 Qureshi AI, Suri MF, Nasar A, He W, Kirmani JF, Divani AA, Prestigiacomo CJ, Low RB: Thrombolysis for ischemic stroke in the United States: data from National Hospital Discharge Survey 1999-2001. Neurosurgery 2005;57:647-654.

-10 Nogueira RG, Liebeskind DS, Sung G, Duckwiler G, Smith WS, MERCI, Multi MERCI Writing Committee: Predictors of good clinical outcomes, mortality, and successful revascularization in patients with acute ischemic stroke undergoing thrombectomy: pooled analysis of the Mechanical Embolus Removal in Cerebral Ischemia (MERCI) and Multi MERCI trials. Stroke 2009;40:3777-3783.

11 Writing Committee of Guidelines of Diagnosis and Treatment of Acute Ischemic Stroke, Neurology Department of Chinese Medical Association Cerebrovascular Disease Study Group: Chinese guidelines of diagnosis and treatment of acute ischemic stroke. Chin J Neurol 2010;43:16-153.

12 Writing Committee of Guidelines of Secondary Prevention of Ischemic Stroke and Transient Ischemic Attack, Neurology Department of Chinese Medical Association Cerebrovascular Disease Study Group: Chinese guidelines of secondary prevention of ischemic stroke and transient ischemic attack. Chin J Neurol 2010;43:154160.

-13 Yadav JS, Wholey MH, Kuntz RE, Fayad P, Katzen BT, Mishkel GJ, Bajwa TK, Whitlow P, Strickman NE, Jaff MR, Popma JJ, Snead DB, Cutlip DE, Firth BG, Ouriel K, Stenting and Angioplasty with Protection in Patients at High Risk for Endarterectomy Investigators: Protected carotid-artery stenting versus endarterectomy in high-risk patients. N Engl J Med 2004;351:1493-1501.

14 Ringleb PA, Allenberg J, Bruckmann H, Eckstein HH, Fraedrich G, Hartmann M, Hennerici M, Jansen O, Klein G, Kunze A, Marx P, Niederkorn K, Schmiedt W, Solymosi L, Stingele R, Zeumer H, Hacke W: 30-day results from the SPACE trial of Stent-Protected Angioplasty versus Carotid Endarterectomy in symptomatic patients: a randomised non-inferiority trial. Lancet 2006;368:1239-1247.

15 Mas JL, Chatellier G, Beyssen B, Branchereau A, Moulin T, Becquemin JP, Larrue V, Lievre M, Leys D, Bonneville JF, Watelet J, Pruvo JP, Albucher JF, Viguier A, Piquet P, Garnier P, Viader F, Touze E, Giroud M, Hosseini H, Pillet JC, Favrole P, Neau JP, Ducrocq X: Endarterectomy versus Angioplasty in Patients with Symptomatic Severe Carotid Stenosis (EVA-3S) trial: results up to 4 years from a randomised, multicentre trial. Lancet Neurol 2008;7:885-892.

16 Brott TG, Hobson RW II, Howard G, Roubin GS, Clark WM, Brooks W, Mackey A, Hill MD, Leimgruber PP, Sheffet AJ, Howard VJ, Moore WS, Voeks JH, Hopkins LN, Cutlip DE, Cohen DJ, Popma JJ, Ferguson RD, Cohen SN, Blackshear JL, Silver FL, Mohr JP, Lal BK, Meschia JF, CREST Investigators: Stenting versus endarterectomy for treatment of carotid-artery stenosis. N Engl J Med 2010;363:11-23.

17 Liu X, Xu G: Endovascular treatments of atherosclerotic carotid diseases in China. Int J Stroke 2010;5:417-420.

18 Henry M, Polydorou A, Henry I, Polydorou AD, Hugel M: Carotid angioplasty and stenting under protection: advantages and drawbacks. Expert Rev Med Devices 2008;5:591-603.

19 Coward LJ, McCabe DJ, Ederle J, Featherstone RL, Clifton A, Brown MM: Long-term outcome after angioplasty and stenting for symptomatic vertebral artery stenosis compared with medical treatment in the Carotid And Vertebral Artery Transluminal Angioplasty Study (CAVATAS): a randomized trial. Stroke 2007;38:15261530 .

-20 Qureshi AI, Feldmann E, Gomez CR, Johnston SC, Kasner SE, Quick DC, Rasmussen PA, Suri MF, Taylor RA, Zaidat 00: Consensus conference on intracranial atherosclerotic disease: rationale, methodology, and results. J Neuroimaging 2009;19(suppl 1):1S-10S.

21 Mori T, Fukuoka M, Kazita K, Mori K: Follow-up study after intracranial percutaneous transluminal cerebral balloon angioplasty. AJNR Am J Neuroradiol 1998;19:1525-1533.

-22 SSYLVIA Study Investigators: Stenting of Symptomatic Atherosclerotic Lesions in the Vertebral or Intracranial Arteries (SSYLVIA): study results. Stroke 2004;35:1388-1392.

-23 Fiorella DJ, Levy EI, Turk AS, Albuquerque FC, Pride GL Jr, Woo HH, Welch BG, Niemann DB, Purdy PD, AagaardKienitz B, Rasmussen PA, Hopkins LN, Masaryk TJ, McDougall CG: Target lesion revascularization after Wingspan: assessment of safety and durability. Stroke 2009;40:106-110.

24 Jiang WJ, Yu W, Du B, Gao F, Cui LY: Outcome of patients with $\geq 70 \%$ symptomatic intracranial stenosis after wingspan stenting. Stroke 2011;42:1971-1975. 